\title{
Atomically Dispersed Ru on Ultrathin Pd Nanoribbons
}

Jingjie Ge ${ }^{\dagger, \Delta}$ Dongsheng He, ${ }^{\ddagger, \Delta}$ Wenxing Chen, ${ }^{\S}$ Huanxin Ju, ${ }^{\&}$ Han Zhang, ${ }^{\dagger}$ Tingting Chao, ${ }^{\dagger}$ Xiaoqian Wang, ${ }^{\dagger}$ Rui You, ${ }^{\dagger}$ Yue Lin, ${ }^{\dagger}$ Yu Wang,${ }^{\prime \prime}$ Junfa Zhu, ${ }^{\text {\& }}$ Hai Li,${ }^{\#}$ Bin Xiao, ${ }^{\dagger}$ Weixin Huang, ${ }^{\dagger}$ Yuen Wu, ${ }^{\dagger}$ Xun Hong, ${ }^{*, \dagger}$ and Yadong $\mathrm{Li}^{*},+, \S$

${ }^{\dagger}$ Center of Advanced Nanocatalysis (CAN) and Department of Chemistry, University of Science and Technology of China, Hefei, Anhui 230026, China

${ }^{\ddagger}$ Materials Characterization and Preparation Center (MCPC), South University of Science and Technology of China, Shenzhen, Guangdong 518055, China

${ }^{\S}$ Department of Chemistry and Collaborative Innovation Center for Nanomaterial Science and Engineering, Tsinghua University, Beijing 100084, China

${ }^{\&}$ National Synchrotron Radiation Laboratory (NSRL), University of Science and Technology of China, Hefei, Anhui 230029, China

"Shanghai Synchrontron Radiation Facilities, Shanghai Institute of Applied Physics, Chinese Academy of Science, Shanghai 201800, China

${ }^{\#}$ Key Laboratory of Flexible Electronics (KLOFE) \& Institute of Advanced Materials (IAM), Jiangsu National Synergetic Innovation Center for Advanced Materials (SICAM), Nanjing Technology University, Nanjing, Jiangsu 211816, China

\section{Experimental Details}

Chemicals. All materials were obtained from commercial suppliers and used without further purification. Palladium(II) 2,4-pentanedionate ( $\mathrm{Pd}(\mathrm{acac})_{2}$, Pd 34.7\%) were purchased from Alfa Aesar. Ruthenium(III) chloride hydrate $\left(\mathrm{RuCl}_{3} \cdot \mathrm{xH}_{2} \mathrm{O}, 99.98 \%\right)$ and allyl benzyl ether $(99 \%)$ was purchased from Aldrich. Poly(vinyl pyrrolidone) (PVP, K30) was purchased from Sinopharm Chemical Reagent Co., Ltd (Ourchem). Methanol (MeOH) was purchased from Sinopharm Chemical Reagent Co., Ltd (Shanghai, China). $N, N$-dimethylacetamide (DMA) $(99.8 \%$, HyDry, with molecular sieves, Water $\leqslant 50 \mathrm{ppm}$ (by K.F.), HySeal) was purchased from HY Scientific Chemical Reagent Co., Ltd. (Shanghai, China). Deionized (DI) water from Milli-Q System (Millipore, Billerica, MA) was used in all our experiments. Commercial $\mathrm{Ru} / \mathrm{C}$ catalyst $(5 \%$ metal, $\sim 1.5 \mathrm{~nm}$ ) was purchased from Aladdin. Commercial $\mathrm{Pd} / \mathrm{C}$ catalyst (10\% metal, $\sim 3.8 \mathrm{~nm}$ ) was purchased from Sinopharm Chemical Reagent Co., Ltd (Ourchem). All regents were used as received without further purification.

Synthesis of the ultrathin Pd nanosheets. The ultrathin Pd nanosheets were synthesized similarly to the previously reported methods ${ }^{1,2}$. In a typical synthesis, Pd(acac) $2(0.032 \mathrm{mmol}, 10.0 \mathrm{mg})$, PVP (32 $\mathrm{mg}$ ) were dissolved in $2 \mathrm{~mL}$ DMA and $4 \mathrm{~mL}$ DI water solution by magnetic stirring under 1 atm atmosphere of $\mathrm{CO}$. The mixture was heated from room temperature to $100^{\circ} \mathrm{C}$ within 20 min and kept for 2 hours under magnetic stirring. After cooling to room temperature, the final dark blue products were 
separated by centrifugation and purified by a DI water-acetone mixture to remove excess PVP molecules.

Synthesis of the ultrathin $\mathbf{P d} / \mathbf{R u}$ nanoribbons. In a typical synthesis, $\operatorname{Pd}(\mathrm{acac})_{2}(0.032 \mathrm{mmol}, 10.0$ $\mathrm{mg}$ ), PVP (32 mg) were dissolved in $2 \mathrm{~mL}$ DMF and $4 \mathrm{~mL}$ DI water solution following under magnetic stirring under $1 \mathrm{~atm} \mathrm{CO}$ atmosphere. The mixture was heated from room temperature to $100^{\circ} \mathrm{C}$ in about $20 \mathrm{~min}$ and kept for 2 hours under magnetic stirring. Then the $\mathrm{RuCl}_{3} \cdot \mathrm{xH}_{2} \mathrm{O}$ solution $(0.24 \mathrm{mmol}$, $62 \mathrm{mg} / \mathrm{mL}, 800 \mu \mathrm{L}$ ) was injected into the mixture solution by pipette and kept stirred at $100^{\circ} \mathrm{C}$ for another 30 minutes. After cooling to room temperature, the final dark blue products were separated by centrifugation and purified by a DI water-acetone mixture to remove excess PVP molecules. To investigate the growth process, the samples were collected respectively, which were treated at $100^{\circ} \mathrm{C}$ for $1,2,5$, 10 and 20 min after the $\mathrm{RuCl}_{3} \cdot \mathrm{xH}_{2} \mathrm{O}$ solution was added into the mixture.

\section{X-ray absorption find spectroscopy (XAFS) measurements and data processing:}

The X-ray absorption fine structure data were collected at BL14W1 station in Shanghai Synchrotron Radiation Facility (SSRF). The electron storage ring of SSRF was operated at $3.5 \mathrm{GeV}$ with a maximum current of $250 \mathrm{~mA}$. The spectra were recorded in the transmission mode for Ru K-edge and Pd K-edge by using $\mathrm{Si}(311)$ double-crystal monochromator, respectively. The XAFS data were collected in ambient conditions. Standard compounds, Ru foil (edge energy 22117eV), Pd foil (edge energy 24350eV) and the sample for energy calibration were tested simultaneously by utilizing the third ionization chamber.

Utilizing the ATHENA module of the IFEFFIT software packages, the obtained EXAFS data were performed according to the standard procedures. The $\mathrm{k}^{2}$-weighted EXAFS spectra were drew through post-edge background subtraction from the overall absorption and normalizing in terms of the edge-

jump step. Subsequently, the EXAFS contributions were separated from different coordination shells by using a hanning windows $\left(\mathrm{d} k=1.0 \AA^{-1}\right)$, then $\mathrm{k}^{2}$-weighted $\chi(\mathrm{k})$ data of both Ru K-edge and Pd K-edge were Fourier transformed to real (R) space. To acquire the quantitative structural parameters around central atoms, least-squares curve parameter was fitted by the ARTEMIS module of the IFEFFIT software packages. ${ }^{3}$

The following EXAFS equation was used:

$$
\chi(k)=\sum_{j} \frac{N_{j} S_{o}^{2} F_{j}(k)}{k R_{j}^{2}} \exp \left[-2 k^{2} \sigma_{j}^{2}\right] \exp \left[\frac{-2 R_{j}}{\lambda(k)}\right] \sin \left[2 k R_{j}+\phi_{j}(k)\right]
$$


$S_{0}{ }^{2}$ is the amplitude reduction factor, $F_{j}(k)$ is the effective curved-wave backscattering amplitude, $N_{j}$ is the number of neighbors in the $j^{\text {th }}$ atomic shell, $R_{j}$ is the distance between the X-ray absorbing central atom and the atoms in the $j^{\text {th }}$ atomic shell (backscatterer), $\lambda$ is the mean free path in $\AA, \sigma_{j}$ is the DebyeWaller parameter of the $j^{\text {th }}$ atomic shell (variation of distances around the average $R_{j}$ ), $\phi_{j}(k)$ is the phase shift (including the phase shift for each shell and the total central atom phase shift). The functions $F_{j}(k)$, $\lambda$ and $\phi_{j}(k)$ were calculated with the ab initio code FEFF8.2. ${ }^{4}$ The additional details for EXAFS simulations are given below.

The coordination numbers of model samples $\left(\mathrm{RuO}_{2}\right.$ and $\mathrm{Pd}$ foil) were fixed as the standard values. The obtained $S_{0}{ }^{2}$ of $\mathrm{RuO}_{2}$ was 0.95 and it was fixed in the subsequent fitting of Ru K-edge data for the $\mathrm{Pd} / \mathrm{Ru}$ nanoribbons. The $S_{0}{ }^{2}$ value of Pd foil was 0.88 and it was also fixed in the subsequent fitting of $\mathrm{Pd} \mathrm{K}$-edge data for the Pd nanosheets and the $\mathrm{Pd} / \mathrm{Ru}$ nanoribbons. While the internal atomic distances $R$, the edge-energy shift $\Delta E_{0}$ and Debye-Waller factor $\sigma^{2}$ were permitted to work separately. The obtained parameters of Pd K-edge were listed in Table S1, and the curve-fitting results were shown in Figure S6, S7 and S8. The obtained parameters of Ru K-edge were listed in Table S2, and the curve-fitting results were shown in Figure S9 and S10.

Catalytic evaluation. All chemoselective hydrogenation reactions were operated with the $25 \mathrm{~mL}$ Schlenk glass vessel tubes under $0.1 \mathrm{MPa}_{2}$ atmosphere. For $O$-benzyl protected monoene, allyl benzyl ether $(0.5 \mathrm{mmol})$ and catalyst $(0.0025 \mathrm{mmol})$ were dispersed in $\mathrm{MeOH}(2.0 \mathrm{~mL})$ under $\mathrm{H}_{2}(1 \mathrm{~atm})$. In the beginning of the reaction, mesitylene $(0.5 \mathrm{mmol})$ as the internal standard was added. Samples were taken to monitor the conversion of allyl benzyl ether and meanwhile to detect the chemoselectivity of benzyl propyl ether by ${ }^{1} \mathrm{H}$ NMR. To reduce the error caused by environment, the catalytic reactions were carried out simultaneously with the $\mathrm{Pd} / \mathrm{Ru}$ nanoribbons, commercial $\mathrm{Pd} / \mathrm{C}$ and commercial $\mathrm{Ru} / \mathrm{C}$ catalysts in room temperature for $1 \mathrm{~h}$. The GC-MS spectrometry and ${ }^{1} \mathrm{H}$ NMR were utilized for the identification and detection of the chemoselective hydrogenation product benzyl propyl ether,. ${ }^{1} \mathrm{H}$ NMR $\left(\mathrm{CDCl}_{3}\right)$ of benzyl propyl ether: $\delta 7.35-7.28(\mathrm{~m}, 5 \mathrm{H}), 4.53(\mathrm{~s}, 2 \mathrm{H}), 3.48(\mathrm{t}, \mathrm{J}=8.8 \mathrm{~Hz}, 2 \mathrm{H}), 1.71-1.62$ $(\mathrm{m}, 2 \mathrm{H}), 0.96(\mathrm{t}, \mathrm{J}=7.2 \mathrm{~Hz}, 3 \mathrm{H}) \mathrm{ppm}^{5}$

Characterization. Transmission electron microscopy (TEM) images of samples were acquired on a Hitachi H-7650 transmission electron microscope at an acceleration voltage of $100 \mathrm{kV}$. Aberrationcorrected High-angle annular dark-field scanning transmission electron microscopy (HAADF-STEM) images and the EDS of samples were performed with a JEOL JEM-2010 LaB6 high-resolution transmission electron microscope operated at $200 \mathrm{kV}$. Inductively coupled plasma and atomic emission spectrum (ICP-AES) was taken on Optima 7300 DV. Atomic force microscopy (AFM) image was captured 
by Dimension ICON with NanoScope V controller (Bruker) in ScanAsyst mode. The X-ray diffraction (XRD) patterns of samples were recorded on a Rigaku Miniflex-600 operating at $40 \mathrm{kV}$ voltage and 15 $\mathrm{mA}$ current with $\mathrm{Cu} \mathrm{Ka}$ radiation $(\mathrm{I}=1.5418 \AA$ ). The synchrotron radiation photoemission spectroscopy (SRPES) spectra were measured with photon energies of $400 \mathrm{eV}, 500 \mathrm{eV}, 600 \mathrm{eV}$ and $1486.6 \mathrm{eV}$ at the BL11U beamline in the National Synchrotron Radiation Laboratory (NSRL) in Hefei, China. The X-ray absorption find structure data were collected at BL14W1 station in Shanghai Synchrotron Radiation Facility (SSRF). The electron storage ring of SSRF was operated at $3.5 \mathrm{GeV}$ with a maximum current of $250 \mathrm{~mA}$. Catalytic performance of chemoselective hydrogenation reactions was analyzed by ${ }^{1} \mathrm{H}$ NMR (recorded on a Bruker $400 \mathrm{MHz}$ spectrometer, chemical shifts are reported in $\delta$ units relative to $\mathrm{CDCl}_{3}$ $\left[{ }^{1} \mathrm{H} \delta=7.26\right]$ ) and GC-MS spectrometry (recorded on an Agilent 7890A GC system and 5975C inert XL MDS with Triple-Axis Detector).
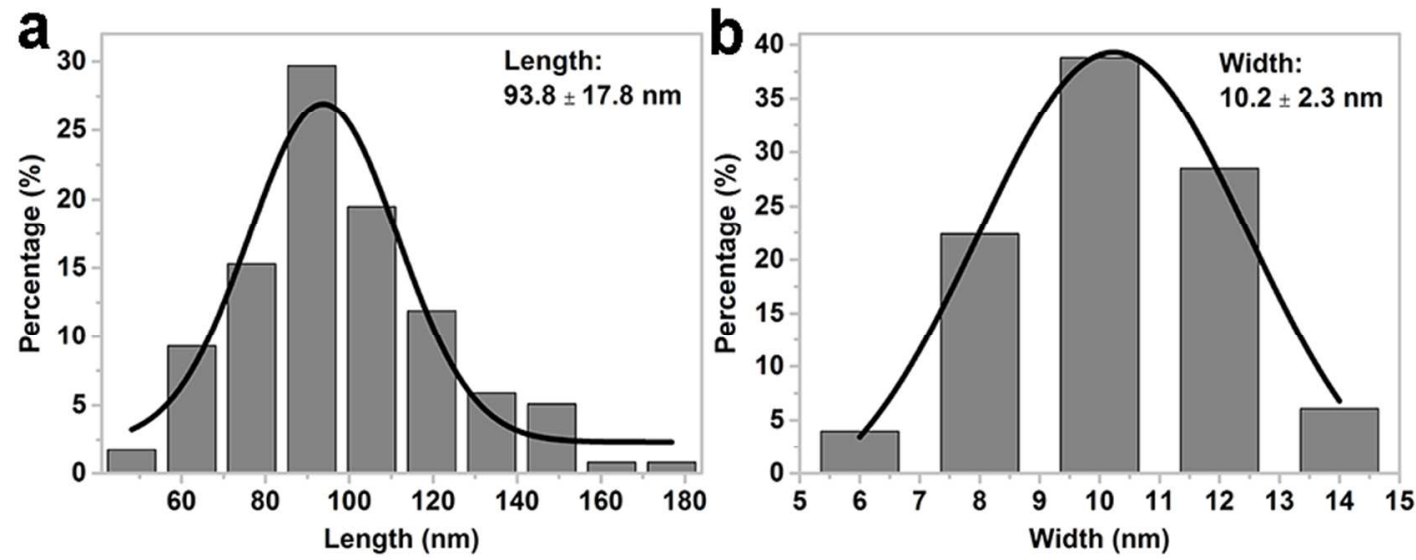

Figure S1. The histograms of (a) length and (b) width distribution of the $\mathrm{Pd} / \mathrm{Ru}$ nanoribbons. Black curve represents Gaussian fit. The ultrathin nanoribbons have an average length and width of $93.8 \mathrm{~nm}$ and $10.2 \mathrm{~nm}$, respectively. 


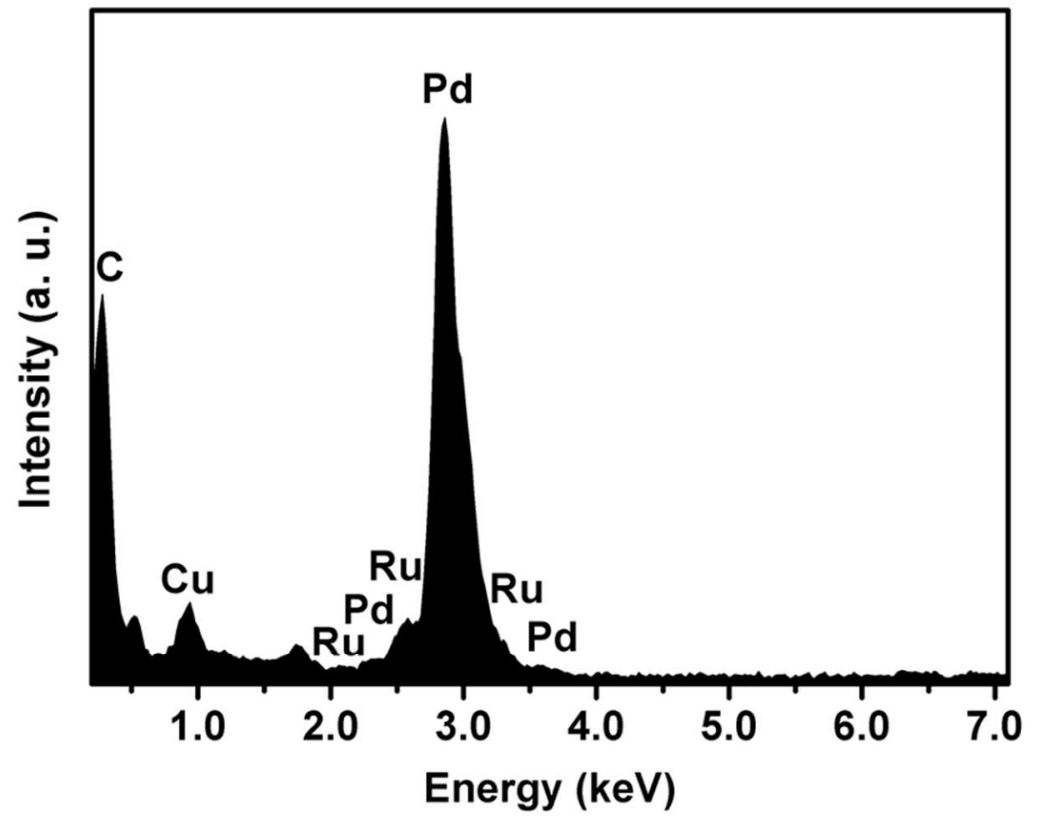

Figure S2. The EDS spectrum of the $\mathrm{Pd} / \mathrm{Ru}$ nanoribbons. The atom ratio of $\mathrm{Ru}$ and $\mathrm{Pd}$ of the ultrathin nanoribbons is about $1: 16$.
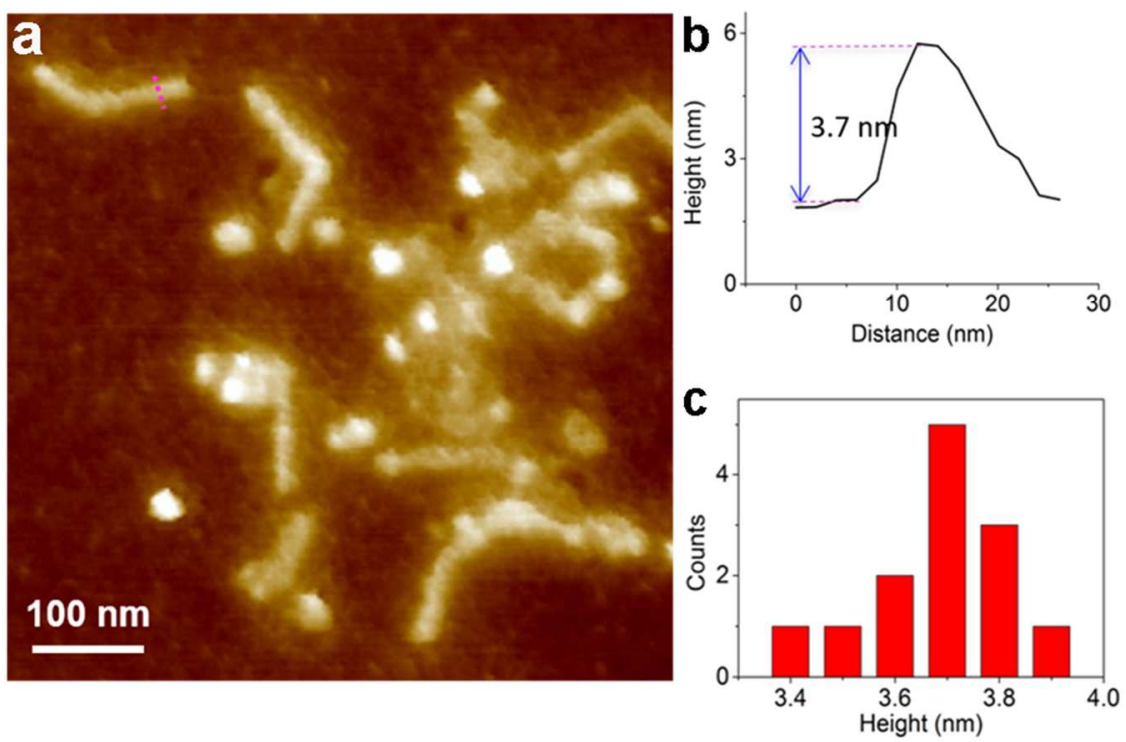

Figure S3. (a) AFM image and (b) the corresponding height profiles of a bare Pd/Ru nanoribbon. (c) The histogram of height distribution of the $\mathrm{Pd} / \mathrm{Ru}$ nanoribbons shown in (a). 


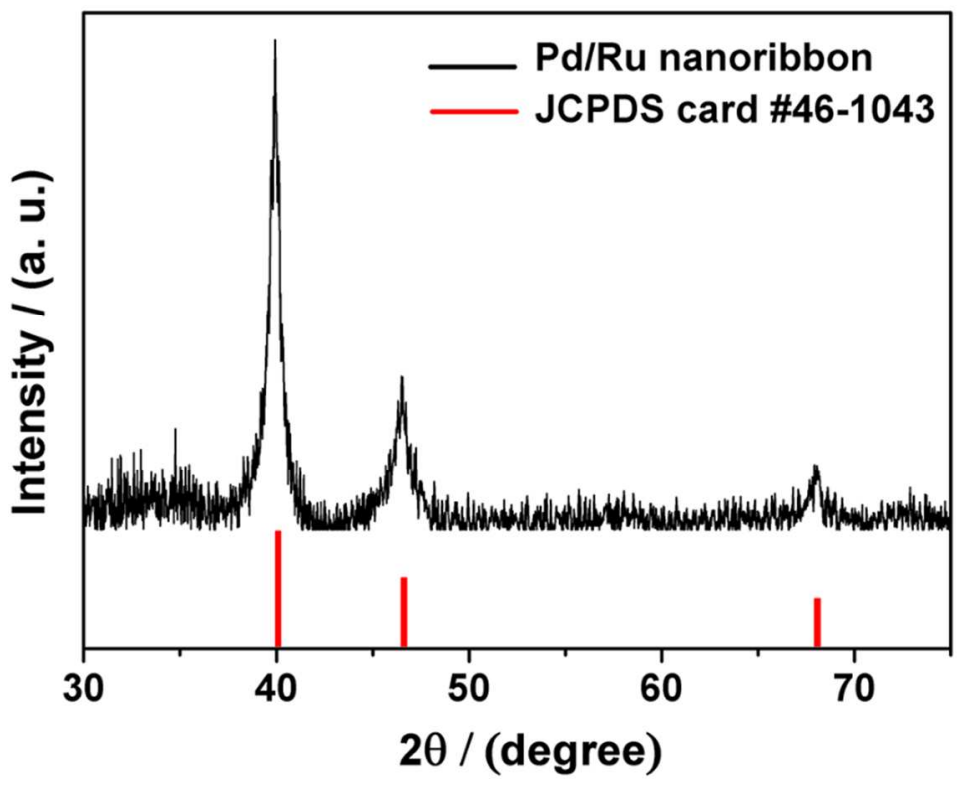

Figure S4. Powder X-ray diffraction (XRD) of the $\mathrm{Pd} / \mathrm{Ru}$ nanoribbons, the $\mathrm{XRD}$ pattern shows three pronounced reflection peaks at $2 \theta=40.01^{\circ}, 46.59^{\circ}$ and $68.09^{\circ}$ are considered to correspond to the fcc $\operatorname{Pd}(111),(200)$ and (220) reflections, respectively.

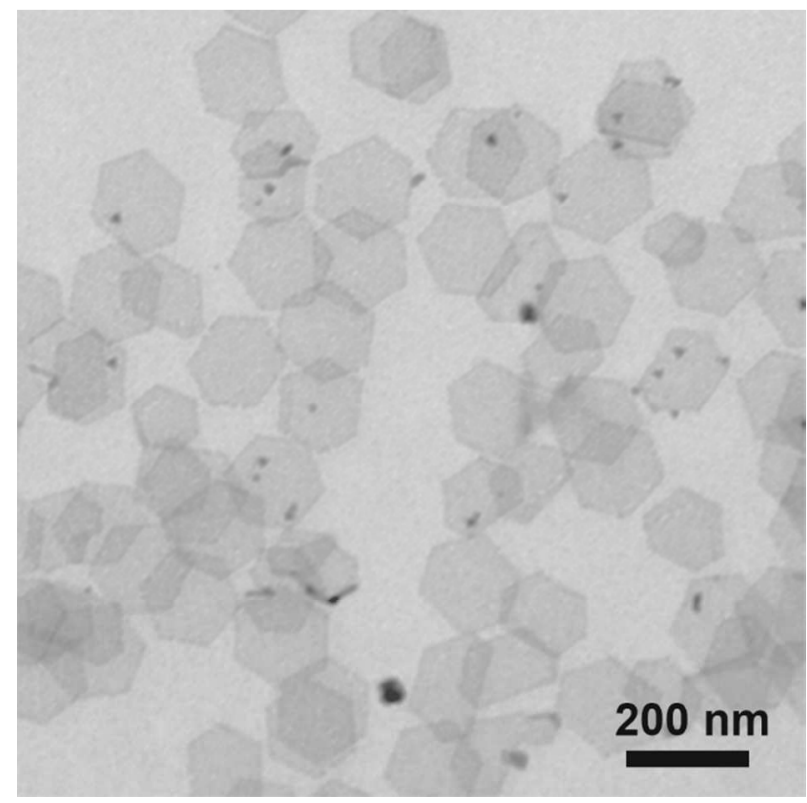

Figure S5. TEM image of the ultrathin Pd nanosheets. 


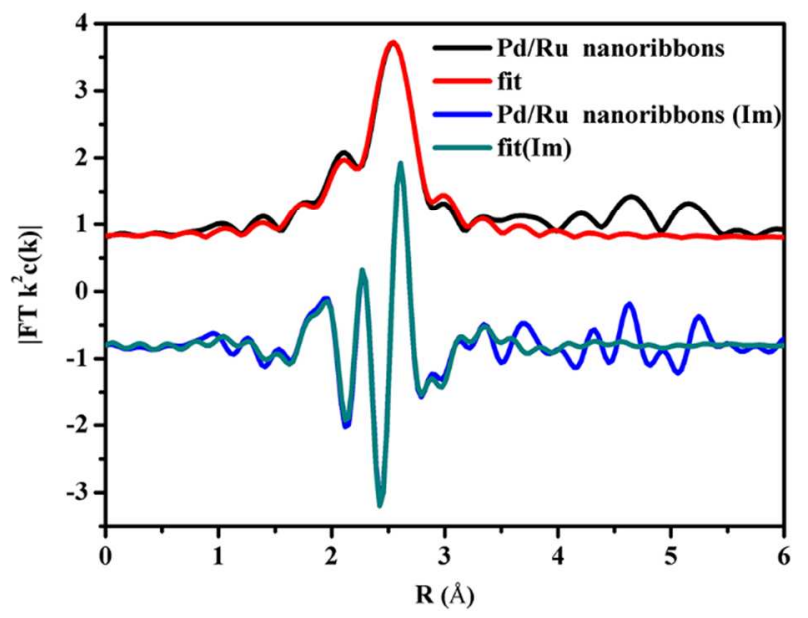

Figure S6. Fitting results of the EXAFS spectra of the Pd/Ru nanoribbons (FT range: $2.94 \AA^{-1}-12.94$ $\AA^{-1}$, Fitting range: $1.58 \AA$ - 3.21 Å.). 


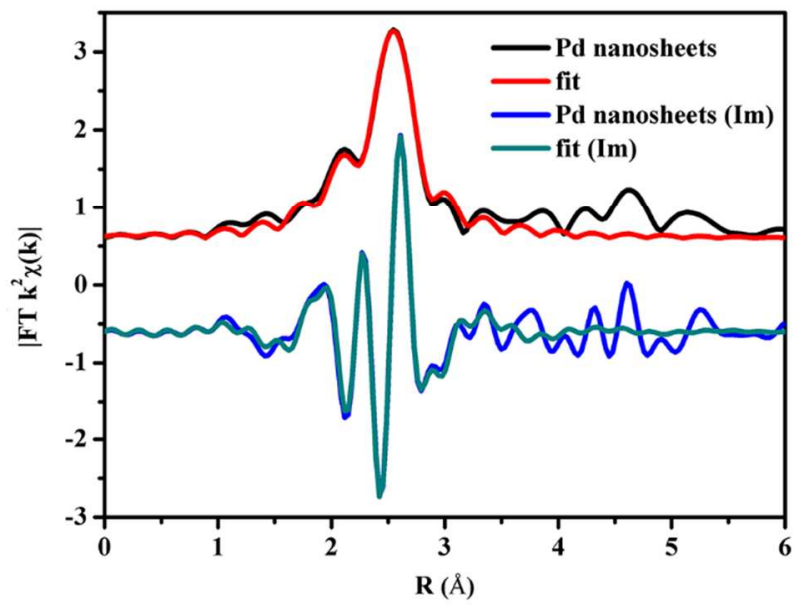

Figure S7. Fitting results of the EXAFS spectra of the Pd nanosheets (FT range: $2.94 \AA^{-1}-12.94 \AA^{-1}$, Fitting range: $1.51 \AA-3.21 \AA$.).

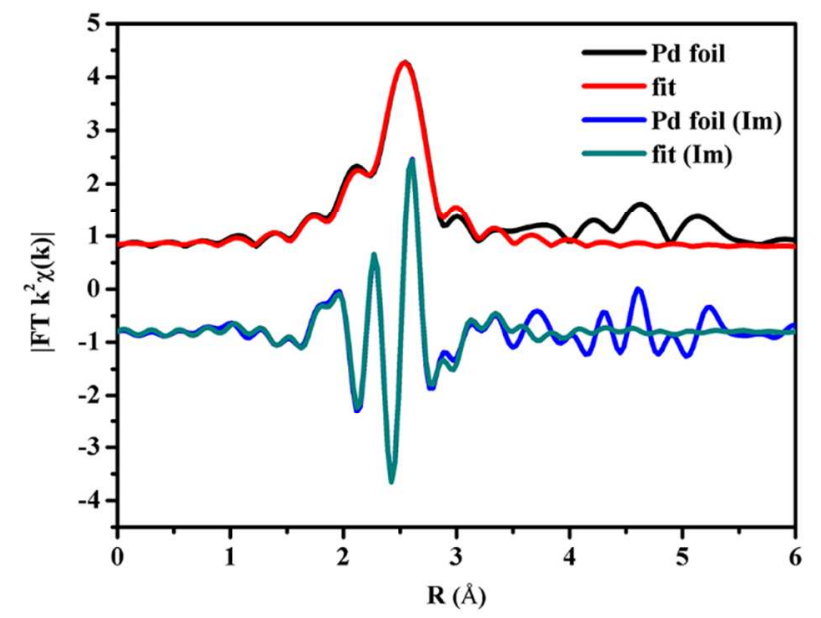

Figure S8. Fitting results of the EXAFS spectra of Pd foil (FT range: $2.94 \AA^{-1}-12.94 \AA^{-1}$, Fitting range: $1.54 \AA$ - $3.21 \AA$ A.). 


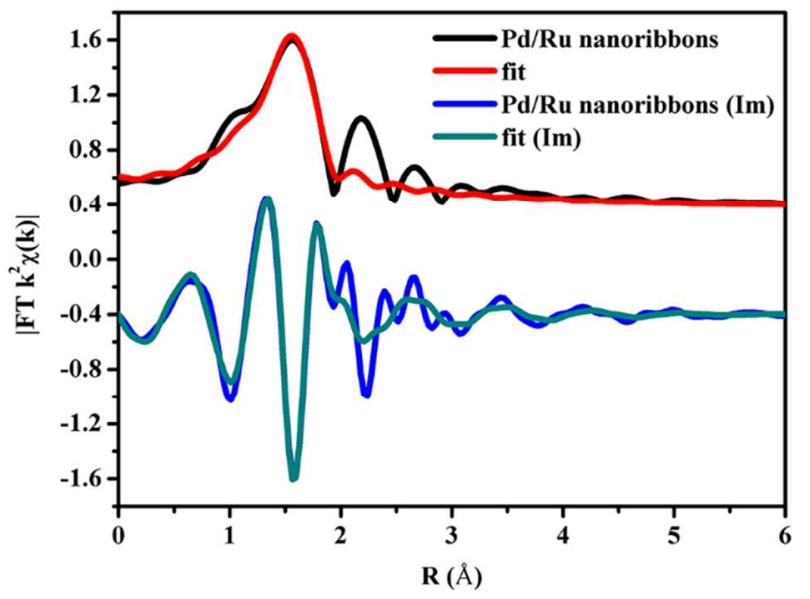

Figure S9. Fitting results of the EXAFS spectra of the Pd/Ru nanoribbons (FT range: $3.88 \AA^{-1}-12.58$ $\AA^{-1}$, Fitting range: $1.20 \AA-1.94 \AA$.).

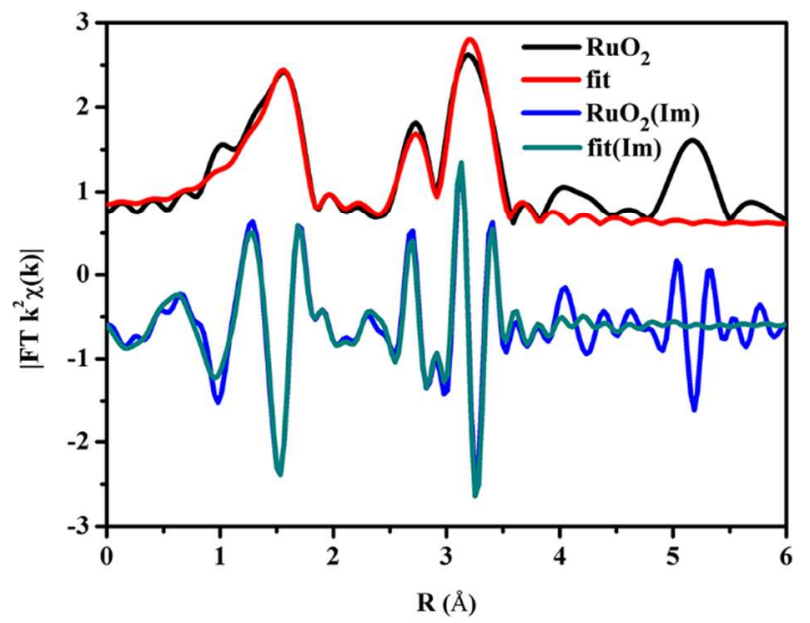

Figure S10. Fitting results of the EXAFS spectra of $\mathrm{RuO}_{2}$ (FT range: $4.06 \AA^{-1}-15.59 \AA^{-1}$, Fitting range: $1.14 \AA \AA-3.60 \AA$.$) .$ 


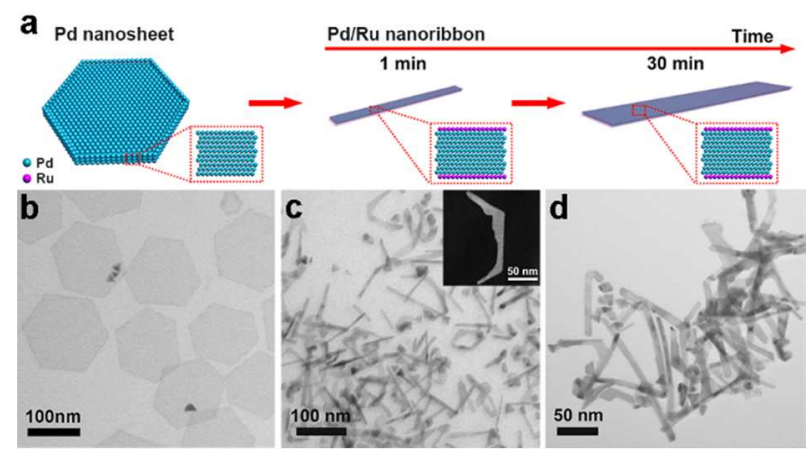

Figure S11. (a) Schematic illustration of the formation of $\mathrm{Pd} / \mathrm{Ru}$ nanoribbon from $\mathrm{Pd}$ nanosheet. (b) TEM image of Pd nanosheet. (c, d) TEM images of the nanoribbons with different reaction time after $\mathrm{RuCl}_{3} \cdot \mathrm{xH}_{2} \mathrm{O}$ are added: (c) $1 \mathrm{~min}$ and (d) $30 \mathrm{~min}$.

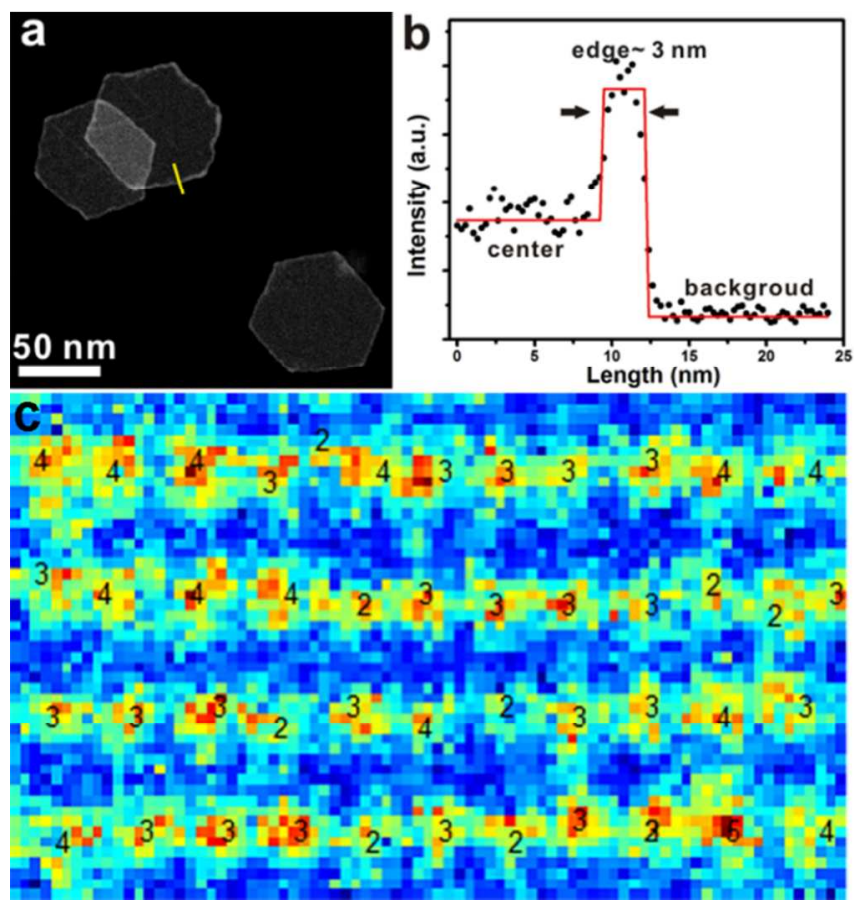

Figure S12. (a) HAADF-STEM image of the ultrathin Pd nanosheets. (b) Line profile of the HAADFSTEM intensity from the region marked by the yellow arrow in panel (a). (c) Atomic resolution aberration-corrected HAADF-STEM images of the center area of a Pd nanosheet. A model-based analysis, which was firstly developed by Van Aert et al, has been applied to this image to estimate the atomic thickness of the atomic columns. ${ }^{6}$ The number of atoms within the atomic column is displayed at the column peak site and it shows that the surface of the Pd was not atomically flat. 

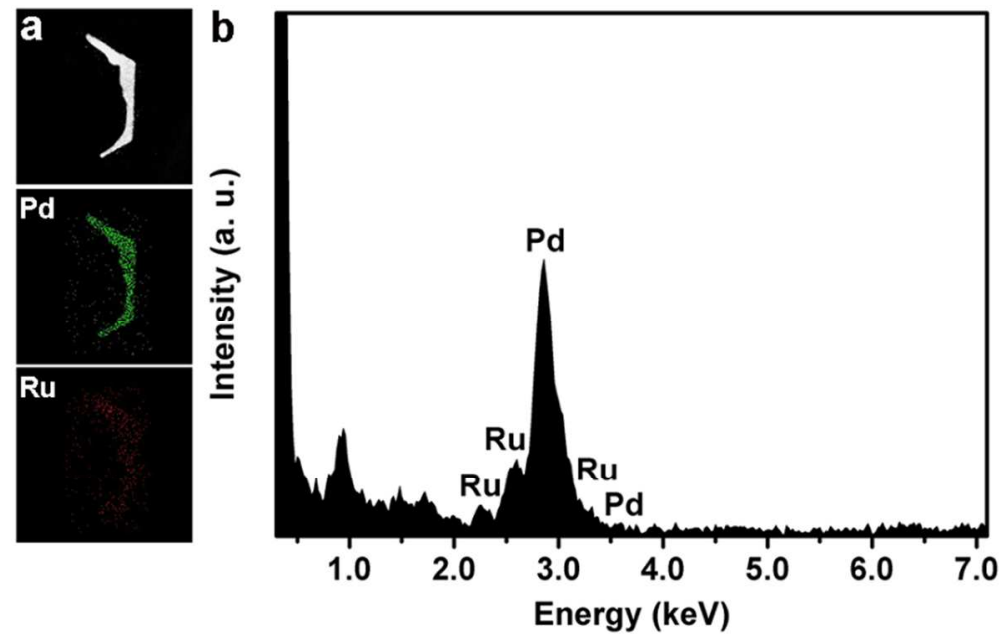

Figure S13. (a) HAADF-STEM image of a kinked Pd/Ru nanoribbon and the corresponding energydispersive X-ray spectroscopy mappings and (b) The EDS spectrum of intermediate products, which confirmed that $\mathrm{Pd} / \mathrm{Ru}$ nanoribbons were formed with a reaction of 1 min after $\mathrm{RuCl}_{3} \cdot \mathrm{xH}_{2} \mathrm{O}$ solution was added. 

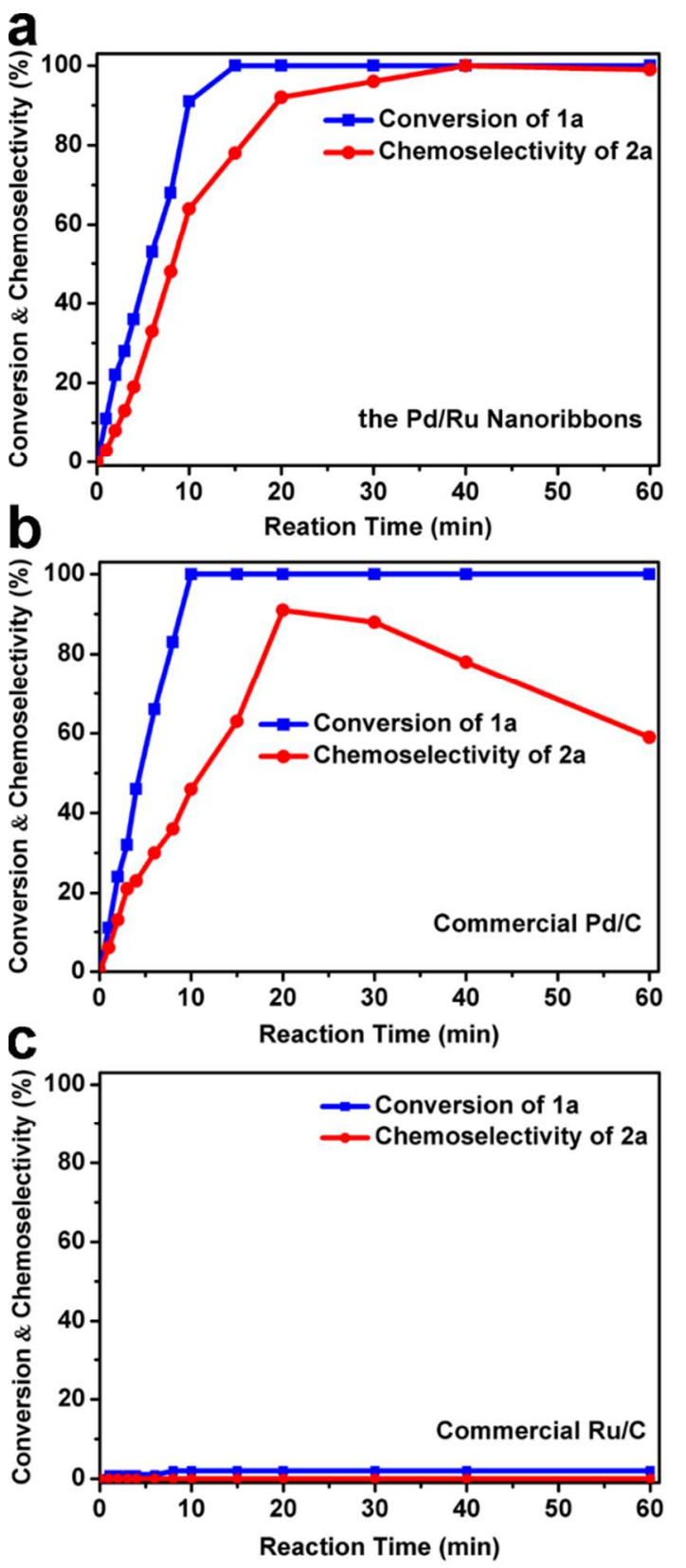

Figure S14. Time-dependent catalytic performance of chemoselective hydrogenation of allyl benzyl ether. Conversion of allyl benzyl ether and chemoselectivity of benzyl propyl ether catalyzed by (a) the ultrathin $\mathrm{Pd} / \mathrm{Ru}$ nanoribbons, (b) commercial $\mathrm{Pd} / \mathrm{C}$ and (c) commercial $\mathrm{Ru} / \mathrm{C}$, respectively. Reaction conditions: allyl benzyl ether $(0.5 \mathrm{mmol})$, catalyst $(0.0025 \mathrm{mmol}), \mathrm{MeOH}(2 \mathrm{~mL}), \mathrm{H}_{2}(1 \mathrm{~atm})$, room temperature, $1 \mathrm{~h}$. Determined by ${ }^{1} \mathrm{H}$ NMR. 


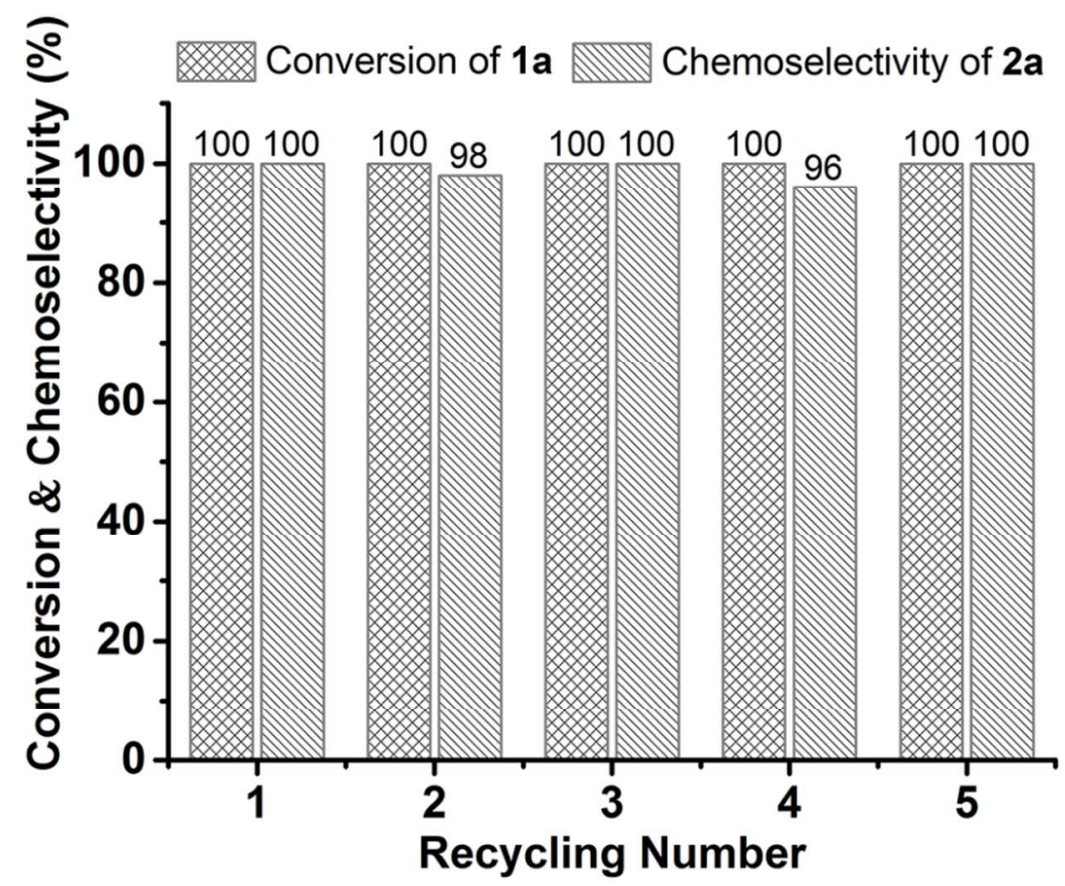

Figure S15. The recyclability of the $\mathrm{Pd} / \mathrm{Ru}$ nanoribbons for the chemoselective hydrogenation of allyl benzyl ether. Reaction conditions: allyl benzyl ether $(0.5 \mathrm{mmol})$, catalyst $(0.0025 \mathrm{mmol}), \mathrm{MeOH}(2 \mathrm{~mL})$, $\mathrm{H}_{2}(1 \mathrm{~atm})$, room temperature, $1 \mathrm{~h}$. Determined by ${ }^{1} \mathrm{H}$ NMR.
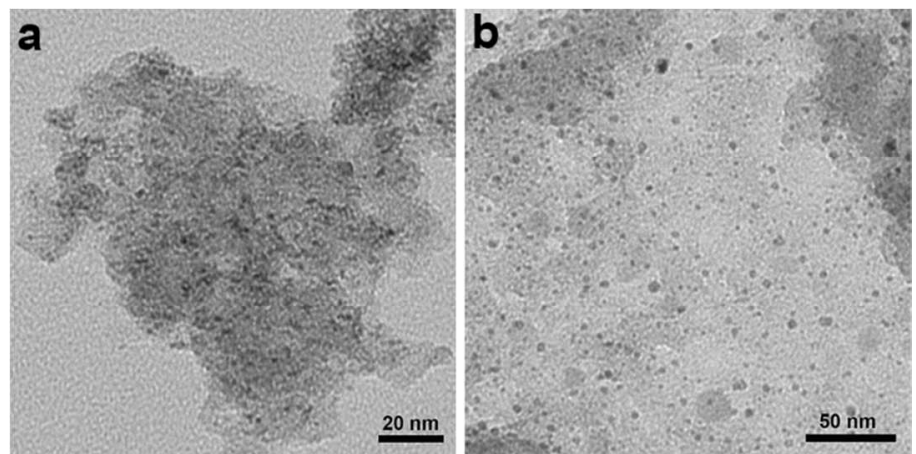

Figure S16. TEM images of (a) commercial $\mathrm{Ru} / \mathrm{C}$ and (b) commercial $\mathrm{Pd} / \mathrm{C}$ catalysts, respectively. 
Table S1 Structural parameters of the $\mathrm{Pd} / \mathrm{Ru}$ nanoribbons, the $\mathrm{Pd}$ nanosheets and $\mathrm{Pd}$ foil extracted from the EXAFS fitting. $\left(\mathrm{S}_{0}{ }^{2}=0.88\right)$

\begin{tabular}{|c|c|c|c|c|c|c|}
\hline Sample & $\begin{array}{c}\text { Scattering } \\
\text { pair }\end{array}$ & $\mathrm{CN}$ & $\mathrm{R}(\AA)$ & $\sigma^{2}\left(10^{-3} \AA^{2}\right)$ & $\Delta \mathrm{E}_{0}(\mathrm{eV})$ & $\mathrm{R}$ factor \\
\hline $\begin{array}{c}\mathrm{Pd} / \mathrm{Ru} \\
\text { nanoribbons }\end{array}$ & $\mathrm{Pd}-\mathrm{Pd}$ & $\begin{array}{c}11.2 \pm \\
0.6\end{array}$ & $\begin{array}{c}2.74 \pm \\
0.03\end{array}$ & $6.5 \pm 0.4$ & $3.1 \pm 0.4$ & 0.002 \\
\hline $\begin{array}{c}\mathrm{Pd} \\
\text { nanosheets }\end{array}$ & $\mathrm{Pd}-\mathrm{Pd}$ & $\begin{array}{c}10.3 \pm \\
0.7\end{array}$ & $\begin{array}{c}2.74 \pm \\
0.03\end{array}$ & $6.6 \pm 0.5$ & $4.6 \pm 0.5$ & 0.003 \\
\hline $\mathrm{Pd}$ foil & $\mathrm{Pd}-\mathrm{Pd}$ & $12 *$ & $\begin{array}{c}2.74 \pm \\
0.03\end{array}$ & $5.7 \pm 0.4$ & $3.8 \pm 0.4$ & 0.002 \\
\hline
\end{tabular}

$\mathrm{CN}$ is the coordination number; $\mathrm{R}$ is interatomic distance; $\sigma^{2}$ is Debye-Waller factor; $\Delta \mathrm{E}_{0}$ is edge-energy shift.

* This value was fixed during EXAFS fitting, based on the known structure of Pd.

Table S2 Structural parameters of the $\mathrm{Pd} / \mathrm{Ru}$ nanoribbons and $\mathrm{RuO}_{2}$ model sample extracted from the EXAFS fitting. $\left(\mathrm{S}_{0}{ }^{2}=0.95\right)$

\begin{tabular}{|c|c|c|c|c|c|c|}
\hline Sample & $\begin{array}{c}\text { Scattering } \\
\text { pair }\end{array}$ & $\mathrm{CN}$ & $\mathrm{R}(\AA)$ & $\sigma^{2}\left(10^{-3} \AA^{2}\right)$ & $\Delta \mathrm{E}_{0}(\mathrm{eV})$ & $\mathrm{R}$ factor \\
\hline $\begin{array}{c}\mathrm{Pd} / \mathrm{Ru} \\
\text { nanoribbons }\end{array}$ & $\mathrm{Ru}-\mathrm{O}$ & $\begin{array}{c}5.2 \pm \\
0.6\end{array}$ & $\begin{array}{c}2.04 \pm \\
0.02\end{array}$ & $5.2 \pm 0.3$ & $-2 \pm 1$ & 0.0013 \\
\hline \multirow{3}{*}{$\mathrm{RuO}_{2}$} & $\mathrm{Ru}-\mathrm{O}$ & $6^{*}$ & $\begin{array}{r}1.98 \pm \\
0.01\end{array}$ & $3.2 \pm 0.3$ & $-2 \pm 1$ & \\
\cline { 2 - 5 } & $\mathrm{Ru}-\mathrm{Ru}$ & $2^{*}$ & $\begin{array}{c}3.09 \pm \\
0.02\end{array}$ & $2.9 \pm 0.7$ & \multirow{2}{*}{$0.10 \pm 4$} & \multirow{2}{*}{0.0093} \\
\cline { 2 - 5 } & $\mathrm{Ru}-\mathrm{Ru}$ & $8^{*}$ & $\begin{array}{c}3.55 \pm \\
0.02\end{array}$ & $3.4 \pm 0.6$ & & \\
\hline
\end{tabular}

$\mathrm{CN}$ is the coordination number; $\mathrm{R}$ is interatomic distance (the bond length between $\mathrm{Ru}$ central atoms and surrounding coordination atoms); $\sigma^{2}$ is Debye-Waller factor (a measure of thermal and static disorder in absorber-scatterer distances); $\Delta \mathrm{E}_{0}$ is edge-energy shift (the difference between the zero kinetic energy value of the sample and that of the theoretical model).

* This value was fixed during EXAFS fitting, based on the known structure of $\mathrm{RuO}_{2}$.

\section{References}

(1) Siril, P. F.; Ramos, L.; Beaunier, P.; Archirel, P.; Etcheberry, A.; Remita, H. Chem. Mater. 2009, 21, 5170 .

(2) Huang, X.; Tang, S.; Mu, X.; Dai, Y.; Chen, G.; Zhou, Z.; Ruan, F.; Yang, Z.; Zheng, N. Nat. Nanotechnol. 2011, 6, 28.

(3) Ravel, B.; Newville, M. J. Synchrotron Radiat. 2005, 12, 537.

(4) Ankudinov, A. L.; Ravel, B.; Rehr, J. J.; Conradson, S. D. Phys. Rev. B 1998, 58, 7565.

(5) Imada, Y.; Iida, H.; Kitagawa, T.; Naota, T. Chem. Eur. J. 2011, 17, 5908.

(6) Van Aert, S.; Batenburg, K. J.; Rossell, M. D.; Erni, R.; Van Tendeloo, G. Nature 2011, 470, 374. 